\title{
50 Differences That Make a Difference: A Compendium of Frequently Confused Term Pairs in Psychology
}

\author{
Scott O. Lilienfeld ${ }^{1,2 *}$, Ava L. Pydych ${ }^{3}$, Steven Jay Lynn ${ }^{4}$, Robert D. Latzman ${ }^{5}$ \\ and Irwin D. Waldman ${ }^{1}$
}

${ }^{1}$ Department of Psychology, Emory University, Atlanta, GA, United States, ${ }^{2}$ Department of Psychology, University of Melbourne, Parkville, VIC, Australia, ${ }^{3}$ Department of Psychology, St. Olaf College, Northfield, MN, United States, ${ }^{4}$ Department of Psychology, Binghamton University, Binghamton, NY, United States, ${ }^{5}$ Department of Psychology, Georgia State University, Atlanta, GA, United States

OPEN ACCESS

Edited by:

Jesus de la Fuente,

University of Almería, Spain

Reviewed by:

Melissa Christine Davis,

Curtin University, Australia Javier Fiz Pérez, Università Europea di Roma,

Italy

*Correspondence: Scott O. Lilienfeld slilien@emory.edu

Specialty section: This article was submitted to Educational Psychology, a section of the journal Frontiers in Education

Received: 06 February 2017 Accepted: 30 June 2017

Published: 20 July 2017

Citation:

Lilienfeld SO, Pydych AL, Lynn SJ, Latzman RD and Waldman ID (2017) 50 Differences That Make a

Difference: A Compendium of Frequently Confused Term

Pairs in Psychology.

Front. Educ. 2:37.

doi: 10.3389/feduc.2017.00037
An adequate understanding of specialized terminology is a prerequisite for the mastery of core concepts across all scientific disciplines, including psychological science. In a previous article (Lilienfeld et al., 2015), we presented an annotated list of 50 widely used psychological terms that should generally be avoided, or at best used judiciously and with qualifications in select cases. Herein, we offer a "sequel" by presenting a list of 50 term pairs in psychology and allied fields (e.g., psychiatry, cultural anthropology, and statistics) that are commonly confused in academic writing, popular writing, or both. We draw these terms from multiple domains of psychology, including Sensation, Perception, Learning, and Memory; Social and Cultural Bases of Behavior; Personality Psychology; Psychopathology; and Research Methodology and Statistics. Many of these terms are frequently confused not merely by beginning psychology students but also by advanced psychology students, psychology instructors, and science journalists. Our list of commonly confused psychological term pairs should hopefully be a modest contribution toward enhancing psychological literacy and critical thinking in psychology more broadly.

Keywords: scientific terminology, specialized vocabulary, bottleneck concepts, frequently confused terms, psychological literacy

All sciences rely on specialized language, or "lingo," and psychology is no exception. Indeed, an adequate understanding of terminology is a prerequisite for the mastery of every scientific discipline's core concepts, because terms are referents for concepts (Cohen, 2012). It is therefore not surprising that instructors in all sciences, including psychological science, typically devote a large amount of time in their courses to ensuring an accurate understanding of their field's terminology (Yager, 1983).

Many psychology students and even some beginning psychology instructors find a good deal of psychological terminology to be daunting and at times bewildering, largely because numerous pairs of terms in this field appear superficially to reflect similar or identical concepts that are in fact substantially different. In other cases, term pairs in psychology refer to cognate concepts that nonetheless differ in subtle but significant ways. A number of these term pairs reflect "bottleneck concepts" in psychology (Gurung and Landrum, 2013). Bottleneck concepts (e.g., reinforcement, discrimination, and short-term memory) are psychological ideas that are deceptively difficult, lulling 
students into a false sense of confidence in their understanding. Unless mastered early on, such concepts can impede the learning of other psychological ideas.

In recent years, numerous scholars in the field of psychology education have underscored the importance of psychological literacy (Boneau, 1990; Cranney and Dunn, 2011; Hulme, 2014) as a key goal for psychology undergraduates and graduate students. Although psychological literacy is a multifaceted concept, one of its core components is a grasp of discipline-relevant terminology, especially "a well-defined vocabulary and basic knowledge of the critical subject matter of psychology" [Roberts et al. (2015), p. 2; see also McGovern et al. (2010)].

In a previous article (Lilienfeld et al., 2015), we presented an annotated list of 50 widely used psychological terms that should generally be avoided, or at best used judiciously and with qualifications in select cases. In that article, we excluded commonly confused term pairs because of space constraints, but promised readers that we would present these terms in a forthcoming article. Here, as promised, we offer a "sequel" of sorts to our previous article by offering a list of 50 term pairs in psychology and allied fields (e.g., psychiatry, cultural anthropology, and statistics) that are commonly confused in scholarly writing, textbook writing, popular writing, or all three.

Although a few authors have generated useful lists of commonly confused word pairs in psychology (e.g., Sternberg and Sternberg, 2010), none of these lists has aimed to be comprehensive or broad in coverage. In addition, virtually all of these lists have focused on term pairs that are confused largely or exclusively by beginning psychology students. In this article, we attempt to fill this gap by presenting commonly confused word pairs across several domains of psychology, including learning, memory, emotion, social psychology, personality, psychopathology, research methodology, and psychometrics. In contrast to previous authors, we emphasize term pairs that are commonly confused not merely by beginning psychology students but also by advanced psychology students, psychology instructors, and science writers. As a consequence, our list should serve as a helpful didactic guide for educators and students in psychology and related fields, as well as for scientists who describe and discuss their research and science journalists who write regularly about psychological topics.

\section{FREQUENTLY CONFUSED PSYCHOLOGICAL TERM PAIRS}

\section{Sensation, Perception, Learning, and Memory}

(1) "Negative reinforcement" versus "punishment." This distinction is familiar to every introductory psychology student, who knows (or at least learned) that negative reinforcement, which involves the withdrawal of a stimulus, increases the likelihood of a previous behavior, whereas punishment, which involves the presentation of a stimulus, decreases the likelihood of a previous behavior (Baron and Galizio, 2006). Nevertheless, this fact has not prevented the misuse of these terms in numerous popular sources and even television shows, including The Big Bang Theory (see https://www.youtube.com/watch?v=LhI5h5JZi-U), not to mention their ubiquitous confusion by generations of undergraduate psychology students (Tauber, 1988). For example, in a news story entitled "British Soccer Players Get Negative Reinforcement," the reporter (Michelson, 2011) described a policy whereby members of a British soccer team were forced to drive around in an old, ugly car for a week following a disappointing showing in a game. In fact, the team management was almost surely punishing, not negatively reinforcing, its poorly performing players. It probably goes without saying that the phrase "punishing reinforcer" (e.g., Gupta and Shukla, 1989) is a whopping oxymoron, at least in behavioral lingo.

(2) "Renewal effect" versus "spontaneous recovery." Both terms describe classical conditioning phenomena that emerge following the extinction of a conditioned response. The renewal effect refers to reinstatement of a previously extinguished conditioned response following a change in the current context of learning. For example, following successful exposure therapy to extinguish fear of spiders in a laboratory setting, individuals may experience a resurgence of this fear when they encounter a spider in another context, such as their patio (Mystkowski et al., 2002). In contrast, spontaneous recovery refers to reinstatement of a previously extinguished conditioned response following the passage of time (Bouton, 2004).

(3) "Sensation" versus "perception." Sensation and perception almost surely fall along a continuum. Nevertheless, sensation is traditionally regarded as the initial stage of detecting raw data from the environment via one or more senses (e.g., vision, audition, and olfaction), whereas perception is traditionally regarded as the later stage of interpreting these raw data and transforming them into meaningful information (Ben-Zeev, 1984).

(4) "Working memory" versus "short-term memory." Although these terms are often used interchangeably (e.g., Veletsianos and Russell, 2014), most contemporary cognitive psychology scholars differentiate between them. Specifically, working memory is typically regarded as an interrelated group of systems for the transient storage and manipulation of information. In contrast, short-term memory is typically regarded as one specific system within the broader working memory apparatus, namely, as a system that serves as a "scratch pad" for keeping information active in memory for a few seconds before it is handed off to other systems for further processing (Baddeley, 1986; Cowan, 2008).

\section{Social and Cultural Bases of Behavior}

(5) "Conformity" versus "obedience." Although both terms refer to forms of social influence, they differ in at least two ways. In conformity, the direction of social influence is "horizontal," that is, from one or more peers to an individual, whereas in obedience, the direction is "vertical," that is, from one or more authority figures to an individual. Moreover, in conformity, the influence is typically implicit (covert), whereas in obedience, it is typically explicit (overt; Loevinger, 1987). For example, whereas the famous Asch (1956) line judgment studies are properly construed as investigations of conformity, the famous Milgram (1963) shock generator studies are properly construed as investigations of obedience. This distinction notwithstanding, some authors refer to Milgram's studies as investigations of conformity (e.g., Wite, 1987). 
(6) "Prejudice" versus "discrimination." Prejudice refers to a belief, whereas discrimination refers to an overt behavior. Specifically, prejudice describes a propensity to "prejudge" others, that is, to arrive at a premature negative judgment of them based on their membership in one or more categories (e.g., AfricanAmerican, Jew, obese, Republican). In contrast, discrimination refers to the act of treating others poorly, such as insulting them, according them fewer resources, or deciding not to hire them, as a function of their membership in one or more categories (Hale, 2002).

(7) "Race" versus "ethnicity." Race refers to a class, such as Caucasian or African-American, that is defined by biological differences, such as white as opposed to brown or black skin. In contrast, ethnicity is a broader concept, such as German or Chinese-American, that not only subsumes race but also encompasses cultural variables, such as country of origin, customs, and preferred language (Schwartz et al., 2016).

(8) "Sex" versus "gender." Admittedly, the distinction here is not universal. Nevertheless, according to the latest edition of the American Psychological Association's (American Psychological Association, 2010) style manual, "sex" is reserved for biological differences, whereas "gender" is reserved for social differences. For example, when referring to men and women in the context of socially defined groups, one should typically use gender, not sex.

\section{Personality Psychology}

(9) "Affect" versus "mood." These two terms are often used in the psychological literature in inconsistent and confusing ways. Nonetheless, in typical psychiatric parlance, and consistent with its meaning in the standard mental status examination, affect is traditionally regarded as a transient and largely specific emotional state, whereas mood is traditionally regarded as a more prolonged and pervasive emotional state (Manjunatha et al., 2008).

(10) "Anxiety" versus "fear." Numerous authors use these terms interchangeably. For example, Wolpe (1987) elected to discuss anxiety and fear synonymously "because they are physiologically indistinguishable" (p. 135). Nevertheless, a consistent body of literature demonstrates that measures of anxiety and fear are weakly or best moderately correlated and display different psychological and physiological correlates (White and Depue, 1999; LeDoux and Pine, 2016). For example, in the brain, anxiety tends to be left lateralized, whereas fear tends to be right lateralized (Sylvers et al., 2011). The bulk of the research literature further suggests that anxiety is associated with negative affect in the presence of an ambiguous and potentially avoidable threat, whereas fear is associated with negative affect in the presence of an imminent and largely unavoidable threat. Moreover, anxiety tends to persist even after threat dissipates, whereas fear tends to diminish or disappear after threat dissipates (Sylvers et al., 2011).

(11) "Empathy" versus "sympathy" Although the construct of empathy appears to be heterogeneous and is often ill defined (Zaki, 2014), most authors define empathy as entailing the capacity to appreciate or grasp the emotions of others. In the eyes of most (e.g., Bloom, 2017) but all not all (e.g., Baron-Cohen, 2011) scholars, the individual experiencing empathy is presumed to experience the same emotions, such as distress, fear, or unhappiness, as is the target of empathy. In contrast, in sympathy, the individual typically experiences concern or compassion for the other person (Clark, 2010; Decety and Michalska, 2010). According to most authors, in empathy, the emotional experience of the two individuals is therefore largely isomorphic, whereas in sympathy, this experience tends to differ considerably.

(12) "Envy" versus "jealousy." These terms are so frequently confused in popular parlance (e.g., "I'm jealous that you're going to Hawaii next week!") that few people are aware that they differ. Nonetheless, the distinction between them is typically straightforward: Envy involves two people, whereas jealousy involves three or more people (Smith and Kim, 2007). For example, the negative emotion a person might experience upon learning that an academic colleague had received a long-sought-after Nobel Prize is envy. In contrast, the negative emotion that this person might experience upon learning that her colleague was invited to a one-on-one dinner by this Nobel Prize winner is jealousy. Hence, you are envious, not jealous, that your friend is headed to Hawaii next week.

(13) "Repression" versus "suppression." In psychoanalytic lingo, repression is a defense mechanism marked by the unconscious motivated forgetting of unpleasant material. In contrast, suppression is a defense mechanism marked by the conscious forgetting of unpleasant material (Akhtar, 2009). Some authors have argued, with reasonable justification, that several widely cited laboratory studies that have purported to provide persuasive evidence for repression (e.g., Levy and Anderson, 2002) in fact provide evidence only for suppression (Kihlstrom, 2002).

(14) "Shame" versus "guilt." Virtually, all scholars concur that shame and guilt differ, although they have not always agreed on the nature of this difference. Most research suggests that shame reflects a global negative evaluation of the self-following a problematic or unethical behavior ("I am bad"), whereas guilt reflects a more specific negative evaluation of this behavior ("I did a bad thing"; Tangney, 1996). In addition, some research suggests that shame tends to be related to avoidance behaviors, whereas guilt tends to be related to approach behaviors, which are intended to redress the harms generated by the action (Schmader and Lickel, 2006).

(15) "Subconscious" versus "unconscious." Freud's biographer Peter Gay (2006) wrote that the tendency to use the term subconscious in lieu of the term unconscious is "a common and telling mistake" (p. 473). Although Freud used the term subconscious in his early writings to refer to the region of the mind that lies immediately below consciousness (e.g., Freud, 1895), he later abandoned his use of this term. Some cognitive psychologists still use this term to describe mental contents that are not presently conscious, but that are potentially accessible to consciousness (e.g., Welsh and Ordóñez, 2014). In this regard, subconscious processing differs from unconscious processing, which is ostensibly inaccessible to awareness.

\section{Psychopathology}

(16) "Antisocial" versus "asocial." Antisocial individuals perform actions against others, as exemplified by individuals who meet criteria for the diagnosis of antisocial personality disorder in the present edition of the American Psychiatric Association's 
(American Psychiatric Association, 2013) Diagnostic and Statistical Manual of Mental Disorders (DSM-5) (Patterson et al., 1992). Such individuals frequently engage in reckless, irresponsible, and at times illegal behaviors. In contrast, asocial individuals chronically withdraw from others (Henle, 2005). They are either shy or disinterested in interpersonal contact. Hence, an article in the Huffington Post, which observed that "the idea that introverts are antisocial or don't want the company of others is completely false" (Gregoire, 2013), conflates this distinction.

(17) "Catalepsy" versus "cataplexy." Both terms refer to potential indicators of mental disorder, but they differ substantially despite their superficial similarity. Catalepsy is a state of muscular rigidity and inflexibility of posture, as observed in schizophrenia with pronounced catatonic features as well as in certain neurological conditions, such Parkinson's disease (Chalasani et al., 2005; Morrison, 2014). In contrast, cataplexy refers to a complete and sudden, but temporary, loss of muscle tone. It is a core feature of the sleep disorder of narcolepsy (Dauvilliers et al., 2007), in which it is frequently triggered by potent emotions. Nevertheless, a number of websites and even some books (McKenzie, 2014) confuse the distinction between these two features.

(18) "Classification" versus "diagnosis." Classification is the act of constructing a system of nomenclature that allows scholars and practitioners to place individuals, organisms, objects, elements, or other entities into categories. For example, the DSM and International Classification of Diseases contain overlapping, although partly competing, classification systems for psychiatric diagnoses. In contrast, diagnosis is the act of assigning individuals to one or more categories within a classification system (Waldman et al., 1995). Hence, the assertion that "undifferentiated-type schizophrenia is a classification used when a person exhibits behaviors which fit into two or more of the other types of schizophrenia" (Ali, 2016) confuses these two terms.

(19) "Delusion" versus "hallucination." Delusions are fixed false beliefs that are not widely shared by members of the individual's culture or subculture, whereas hallucinations are perceptual experiences that occur in the absence of any sensory stimulation (American Psychiatric Association, 2013). These terms are widely confused in popular culture and are occasionally confused in the peer-reviewed literature as well; for example, one author team wrote that "One of the underlying mechanisms of hallucinations of persecution is to misinterpret the intentions of others and to believe in the associative nature of unrelated facts" (Landgraf et al., 2011, p. 8). Because delusions are erroneous beliefs, the authors presumably intended to refer to delusions, not hallucinations. Much of the confusion between these two concepts probably stems from their frequent cooccurrence. For example, many patients who hear persecutory voices (an auditory hallucination) develop the conviction that other people are intending to harm them (a delusion). Also commonly confused with hallucinations are illusions, which are misinterpretations of actual stimuli (Kölmel, 1993).

(20) "Obsession" versus "compulsion." As the latest edition of the DSM (American Psychiatric Association, 2013) notes, obsessions are "recurrent and persistent thoughts urges or images that are experienced as intrusive or unwanted" (p. 235). In contrast, compulsions are "repetitive behaviors or mental acts that an individual feels driven to perform in response to an obsession or according to rules that must be applied rigidly" (p. 235). For example, recurrent thoughts of potential contamination are obsessions, whereas recurrent handwashing intended to neutralize or reduce the frequency of these thoughts are compulsions (Hamstra, 1995). Obsessions are anxiety producing, whereas compulsions are anxiety reducing, at least in the short term.

(21) "Psychopathy" versus "sociopathy." Psychopathy, otherwise known as psychopathic personality, is a personality disorder characterized by a paradoxical combination of features: superficial charm, poise, and low anxiety on the one hand, conjoined with guiltlessness, callousness, dishonesty, and poor impulse control, on the other (Cleckley, 1941/1988; Hare, 1991/2003; Lilienfeld, 1994). "Sociopathy," in contrast, is a colloquial term that refers variously to any one or more of four concepts. Specifically, various authors describe sociopathy as a condition that is (a) synonymous with psychopathy (Mealey, 1995), (b) similar to psychopathy, but characterized by especially poor emotion regulation (Siciliano, 2014), (c) marked by chronic antisocial and criminal behavior that is primarily sociocultural in origin (Partridge, 1930; Lykken, 1995), or (d) marked by a long-standing history of antisocial and criminal behavior dating back at least to adolescence (Robins, 1966), that is, as a condition more or less synonymous with the DSM diagnosis of antisocial personality disorder (American Psychiatric Association, 2013). As Berg et al. (2013) noted, "sociopathy is not a formal psychiatric or psychological term, and its indiscriminate use appears to have engendered little more than conceptual confusion" (p. 66). We therefore recommend its belated retirement (cf., Pemment, 2013).

(22) "Psychosomatic" versus "somatoform." In psychosomatic conditions, individuals experience actual physical symptoms that are assumed by some scholars to be influenced by psychological factors. For example, some skin conditions, such as psoriasis, and some intestinal conditions, such as irritable bowel syndrome, are regarded by many authors as at least partly psychosomatic, because their clinical expression and course appear to be influenced by life stressors (e.g., Rieder and Tausk, 2012). In contrast, in somatoform conditions, such as conversion disorder, individuals are preoccupied with physical symptoms that lack a clear-cut medical explanation (Mayou, 2014). Nevertheless, the term "somatoform" - and its accompanying assumption of a lack of clear-cut medical explanation-was jettisoned from the latest edition of the DSM (American Psychiatric Association, 2013) and replaced by the unwieldy phrase "somatic symptom and related disorders." This new category, which emphasizes excessive physical concerns regarding putative medical symptoms rather than medically unexplained symptoms, has attracted criticism from scholars concerned that it will expand the scope of psychopathology to subsume largely normative reactions to physical problems (e.g., Frances, 2013).

(23) "Schizophrenia" versus "multiple personality disorder" (dissociative identity disorder). Perhaps this distinction is too well known to bear repeating, but its omnipresent misuse in popular culture (see Duckworth et al., 2003, and Lilienfeld et al., 2009 , for a plethora of examples) suggests that a friendly reminder may be in order. For example, as Hacking (1995) observed, in the eyes of the general public, the following formula holds: "multiple 
personality $=$ split personality $=$ schizophrenia" (p. 9). As the great Swiss psychiatrist Eugen Bleuler (1911) observed, schizophrenia is characterized by a severe splitting of functions, such as cognition, emotion, and motivation, within a single person. In contrast, in multiple personality disorder, now termed dissociative identity disorder (American Psychiatric Association, 2013), the individual's mind ostensibly harbors two or more distinct "alters," that is, personalities, or "personality states." It should be noted, however, that many scholars have questioned whether dissociative identity disorder is genuinely characterized by the simultaneous coexistence of multiple distinct alters within an individual (Lilienfeld and Lynn, 2014).

(24) "Serial killer" versus "mass murderer." Shortly before notorious killer Theodore (Ted) Bundy's execution, a reporter referred to him as a "mass murderer" (Keller, 1989). In fact, Bundy was a serial killer, not a mass murderer. The term "serial killer" refers to someone who kills multiple people in a string of incidents that are separated by "cooling off" periods. In contrast, a "mass murderer," such as the 2007 Virginia Tech killer Seung-Hui Cho or the 2012 Aurora, Colorado movie theater killer James Holmes, massacres a large number of people in a single incident (Fox and Levin, 1998). Serial killers, incidentally, should be distinguished from spree killers, whose homicidal episodes are not separated by clear-cut cooling-off periods.

(25) "Symptom" versus "sign." Symptoms and signs are both indicators of psychopathology. Nevertheless, symptoms are subjective and must be reported by patients, whereas signs are largely objective and can be observed by clinicians and others (Lilienfeld et al., 2016; see also Lilienfeld et al., 2015). For example, fatigue is a common symptom of major depressive disorder, whereas psychomotor retardation (slowing of movement) is a common sign of this condition.

(26) "Tangentiality" versus "circumstantiality." Psychiatrists familiar with the mental status exam frequently find the distinction between these terms confusing. In tangentiality, the patient goes off topic (that is, goes off on a "tangent") in response to a question and never returns, frequently leaping from one unrelated topic to another. In contrast, in circumstantiality, the patient more or less remains on topic but veers into somewhat unrelated and often irrelevant details, eventually returning to the original topic (Patel et al., 2006). In tangentiality, the person never reaches the goal (answering the question); in circumstantiality, the person reaches the goal but does so in a highly indirect, inefficient fashion (as mnemonics for the latter term, think of the words "circle" or "circuitous").

(27) “Transgender" versus "transvestite." A transgender person possesses a gender identity that differs from his or her biological sex (when such a person seeks medical intervention to transition to a different sex, he or she is commonly referred to as transsexual). In contrast, a transvestite is someone who dresses in clothing that differs from that traditionally worn by members of his or her biological sex. Nevertheless, many people regard the term "transvestite" to be stigmatizing and outmoded, so the term "cross-dresser" is now generally preferred (Centers for Educational Justice and Community Engagement, 2016). The DSM-5 (American Psychiatric Association, 2013) diagnosis of "transvestic disorder," formerly called transvestic fetishism, is applied to individuals, virtually all of whom are male, who (a) display repeated and intense urges, fantasies, or behaviors relevant to cross dressing and (b) experience clinically significant distress, impairment, or both as a consequence. In contrast to most transgender individuals, the principal attraction of individuals with transvestic fetishism is to people of the opposite biological sex.

\section{Research Methodology and Statistics}

(28) "Cronbach's alpha" versus "homogeneity." Cronbach's alpha (Cronbach, 1951) is the most widely used measure of internal consistency in psychology, psychiatry, and allied fields. This statistic is useful for ascertaining the extent to which a psychometrically parallel set of items constituting a test would yield comparable results in another sample. As a consequence, Cronbach's alpha bears on the stability of a measure. Many authors erroneously believe, however, that Cronbach's alpha is a good metric of a test's homogeneity. For example, when describing the internal consistency of a set of indicators of pain, one author team referred to a "Cronbach's alpha homogeneity coefficient" (BenDebba et al., 2000, p. 91) of 0.82. Nevertheless, statisticians have long recognized that Cronbach's alpha is typically a poor barometer of homogeneity, because it is substantially affected by test length (Cortina, 1993; Sijtsma, 2009). Even when average item intercorrelations are low, Cronbach's alpha will typically be high given a sufficiently large number of items.

(29) "Discriminant validity" versus "discriminative validity." Discriminant validity refers to the extent to which a measure is unrelated, or largely unrelated, to measures of constructs with which it theoretically unassociated (Campbell and Fiske, 1959; Cole, 1987). Nevertheless, many authors mistakenly invoke the term "discriminant validity" to refer to the capacity of a test to discriminate between two groups, such as individuals with schizophrenia versus individuals with no disorder, men versus women, or violent-prone versus non-violent-prone individuals (e.g., McCann et al., 2000). Such usage is incorrect, as the capacity of a test to differentiate between groups is actually an indicator of its convergent validity (Lilienfeld, 2004). The proper term to describe a test's capacity to distinguish between two groups in accord with theoretical prediction is discriminative, not discriminant, validity (Haynes et al., 2011).

(30) "External validity" versus "ecological validity." External validity refers to the extent to which a study's findings are generalizable to other settings, especially those in the real world. For example, psychologists continue to debate the question of whether laboratory results derived from social or cognitive psychological paradigms, such as laboratory studies of aggression (e.g., the Taylor Aggression Paradigm), display external validity with respect to naturalistic environments (e.g., Tedeschi and Quigley, 1996; Anderson and Bushman, 1997). In contrast, ecological validity refers to the extent to which a study design mirrors real-world settings. For example, in contrast to a laboratory study of aggression involving a shock generator, a study of aggression in naturally occurring settings, such as an observational study of barroom brawls (e.g., Graham and Wells, 2003), possesses high levels of ecological validity. Nevertheless, such a study would not possess high external validity if its findings did not generalize 
to other real-world situations (see Mook, 1983, for a broader discussion).

(31) "Face validity" versus "content validity." Face validity refers to the extent to which items on a test appear to be assessing the domain of interest (Lynn, 1986). According to many psychometricians, the term "face validity" is a misnomer, as the concept to which it refers is not technically a form of validity at all (Nevo, 1985). Moreover, face validity hinges in part on the subjective perception of the observer; what may be face valid to one respondent may be face invalid to another. In contrast to face validity, content validity refers to the extent to which the items on a test adequately sample from the universe of the construct domain; a content valid test adequately reflects the full range of the construct (Haynes et al., 1995). In principle, a test can be face valid yet low in content validity and vice versa.

(32) "Factor analysis" versus "principal components analysis." As we and many others have noted elsewhere (e.g., Lilienfeld et al., 2015), factor analysis is designed to undercover the dimensions underlying a correlation matrix. It aims to ascertain broader dimensions that account for the covariation among variables. In contrast, principal components analysis (which is frequently misspelled as "principle components analysis") is designed to create a smaller set of weighted variables (variates) that adequately reproduces most of the variance of the original variables in the dataset. As a consequence, principal components analysis strives to simplify a large set of variables into a more manageable number of variables that capture the bulk of their original variance, most commonly to minimize the risk of Type I error in subsequent analyses (Fabrigar et al., 1999). Whereas factor analysis relies only on the shared variance among variables, principal components analysis preserves all of the variance in these variables.

(33) "Predictive validity" versus "concurrent validity." Many authors use the term "predictive validity" to refer to the extent to which a test correlates with other measures administered at the same time (e.g., Bell et al., 1992). Nevertheless, this term should be reserved for cases in which investigators examine the extent to which a test forecasts future outcomes (Carmines and Zeller, 1979). The error of confusing predictive and concurrent validity probably stems from the confusion between "statistical prediction," which refers to the extent to which one variable is statistically associated with another variable (Meehl, 1954), with longitudinal prediction. Predictive validity refers only to the latter meaning, whereas concurrent validity refers to the extent to which a test correlates with other measures administered at the same time. Both terms are often subsumed under the broader umbrella of "convergent validity."

(34) "Mediator" versus "moderator." The bane of many beginning graduate students' existence, these two terms are readily and widely confused. A mediator is a variable, $C$, that intervenes between two correlated variables $\mathrm{A}$ and $\mathrm{B}$, and that accounts at least in part (partial mediation) or entirely (full mediation) for their statistical association. For example, Starr et al. (2014) found that low sociability and interpersonal oversensitivity mediated the relation between social anxiety disorder among women assessed at age 15 and depression assessed eight years later. In contrast, a moderator is a variable, $\mathrm{C}$, that statistically affects the direction, magnitude, or both, of the relation between variables $\mathrm{A}$ and B. For example, Stern et al. (1982) reported that the positive relation between life events and severity of illness was stronger for uncontrollable events, such as the death of a spouse, than for controllable events, such as divorce. Mediators tend to address "how" or "why" questions (e.g., how does behavioral activation improve the symptoms of depression?), whereas moderators tend to address "when," "for whom," and "under which" questions (e.g., for which gender is the correlation between impulsivity and violence larger? under which conditions is psychopathy most predictive of violence? Baron and Kenny, 1986; Frazier et al., 2004). Making matters more confusing, a variable can in principle be both a mediator and a moderator (James and Brett, 1984).

(35) "Prevalence" versus "incidence." Prevalence refers to the proportion of individuals in a population with a given condition, such as a psychiatric disorder. For example, point prevalence refers to the percentage of people in a population who meet criteria for a given condition at a given moment in time. In contrast, incidence refers to the rate of emergence of new cases of individuals with a condition over a specified time interval, such as 1 month or 1 year (Hoek and Van Hoeken, 2003). More technically, prevalence equals incidence multiplied by duration (Streiner, 1998). This distinction notwithstanding, many authors refer erroneously to the "incidence" of schizophrenia and other mental disorders when they actually intend to refer to prevalence (e.g., Weiser et al., 2002).

(36) "Risk factor" versus "cause." A risk factor is a variable that (a) precedes the onset of a disorder and (b) is associated with a heightened likelihood of developing this disorder. Nevertheless, not all risk factors are causal risk factors (see Kraemer et al., 1997, for a discussion). For example, although attentional dysfunction often precedes the onset of schizophrenia and is statistically associated with this condition (Cornblatt and Obuchowski, 1997), it may merely be an indirect proxy of the etiological processes underlying schizophrenia rather than a contributor to schizophrenia per se. In contrast, the death of a loved one appears to both precede, and be causally related to risk for, the onset of major depression (Kendler, 2005).

(37) "Standard deviation" versus "standard error." The standard deviation is a commonly used index of the dispersion (variability) of scores on a variable; it measures the extent to which scores are "spread out" around the mean. In contrast, the standard error is the standard deviation of the sampling distribution of a statistic, such as the mean or correlation coefficient. Accordingly, it is an index of the certainty of one's estimate of a statistic in one's sample, and it is commonly used to construct confidence intervals surrounding this statistic (Streiner, 1996).

(38) "Stepwise regression" versus "hierarchical regression." The confusion between these terms has contributed to all manner of mischief in the interpretation of multiple regression analyses. In stepwise multiple regression, each predictor variable is selected automatically on the basis of its highest residual (semi-partial) correlation with the outcome variable. In contrast, in hierarchical multiple regression, predictor variables are force entered by the researcher to ascertain their incremental contribution above and beyond other predictor variables. Stepwise regression procedures are notorious for yielding high rates of Type I errors (false positives), because predictor variables may be included or excluded 
from regression equations on the basis of small, even miniscule, differences in their semi-partial correlations. These differences are typically unreplicable across samples (Whittingham et al., 2006; Streiner, 2013). The confusion between stepwise and hierarchical multiple regressions are understandable, because in hierarchical regression the researcher enters the variables in sequential steps.

\section{Miscellaneous}

(39) "Clairvoyance" versus "precognition." The term "clairvoyance" (its etymology derives from the French for "clear vision") is frequently used in popular and scholarly writings to refer to the purported ability to foretell the future by means of extrasensory capacities (e.g., Van Zandwijk and Van De Vijver, 2007; Konnikova, 2016). For example, when describing a study of the role of midbrain dopamine systems in the prediction of future stimuli, one university press release described it as an investigation of "Everyday clairvoyance: How your brain makes decisions" (ScienceDaily, 2011). This usage is incorrect, as clairvoyance is the ostensible ability to detect the presence of hidden objects (or objects otherwise out of sensory contact) by means of psychic powers. The correct term describing the supposed ability to predict the future by means of psychic powers is precognition (Steinkamp, 1999). It should be noted, however, that the scientific support for both clairvoyance and precognition is minimal (Hyman, 2017).

(40) "Coma" versus "persistent vegetative state." The terms coma and persistent vegetative state (PVS; recently renamed unresponsive wakefulness syndrome; Laureys et al., 2010) are closely related but should be distinguished. People who experience a coma following traumatic brain injury, drug overdose or misuse, lack of oxygen to the brain, or illness are unable to respond to external stimulation, such as light or sound; cannot be awakened, respond to verbal commands, or initiate purposeful actions; and lack a normal sleep-wake cycle (Monti et al., 2010). Some people with severe brain damage transition from a coma to a PVS. In a PVS, normal awareness of the self and environment is absent. In contrast to a coma, which typically lasts less than a month, after which the person emerges with no or varying degrees of brain damage, a PVS can last for many years and cause permanent cognitive and functional disability. In contrast to a coma, in a PVS, the person's level of consciousness may vary in response to stimulation, and certain reflexes and automatic responses (e.g., yawning, grimacing, moaning, and opening eyes during feeding) are preserved (Laureys et al., 2004). Although the sleep-wake cycle in PVS appears to persist, with occasional eye opening and closing, it is unclear whether circadian rhythms persist. People sometimes confuse a PVS with brain or cortical death, in which no brain activity or brainstem reflexes are evident. Nevertheless, in a PVS, non-cognitive or lower brain functions, such as breathing, digestion, and circulation, are apparent, and some "islands" of awareness may remain intact (Owen et al., 2006). An irreversible state between a PVS and brain death can pose particularly vexing ethical dilemmas for caretakers when no legal directives exist regarding whether to prolong life through artificial means, as in the highly publicized case of Terry Schiavo, the Florida woman who remained in a PVS for 15 years before being removed from life support in 2005.
(41) "Culture-fair test" versus "culture-free" test. Scores on a culture-fair test display no slope bias (differential validity) across cultures. That is, a culture-fair test is equally associated with the tested ability, such as intelligence, regardless of the culture of the examinee. In contrast, scores on a culture-free test, which is a hypothetical measure that is almost certainly unrealistic as a psychometric goal, are entirely unaffected by the cultural experiences of examinees (Sternberg and Sternberg, 2010).

(42) "Delirium" versus "dementia." The terms delirium and dementia can be easily confused because people with dementia may experience states of delirium marked by changes in consciousness and behavior that include confusion and disorganized thinking, agitation, and alterations of attention, mood, and perception. Delirium often has an abrupt onset and can fluctuate over brief time periods. In contrast, dementias, such as Alzheimer's disease, are typically marked by a slow and progressive onset, with deterioration in memory, reasoning, personality, and psychological and motor functioning developing over months and years (Lippmann and Perugula, 2016). There are many potential causes of delirium in addition to dementia, including infections, alcohol withdrawal, metabolic and electrolyte disturbances, and disorders of the brain, heart, kidney, liver, and lung (Lipowski, 1990). Scientists are still attempting to pinpoint the cause of Alzheimer's disease, although other types of dementia (Chiu et al., 2006) are caused by cardiovascular disease (i.e., vascular dementia), and problems with Lewy bodies/ abnormal proteins (i.e., Lewy body dementia) in the brain and loss of neurons in the frontal and temporal lobes of the brain (i.e., frontotemporal dementia). Episodes of delirium are often treatable, whereas there are no effective long-term interventions to reverse the symptoms of Alzheimer's disease and other dementias (Sink et al., 2005).

(43) "Disease" versus "illness." Most sociologists and cultural anthropologists distinguish disease from illness, with the former being the specific pathology or malfunctioning of a body part, and the latter comprising the afflicted individual's psychological reactions to the disease (Kleinman et al., 2006).

(44) "Flooding" versus "implosion." Flooding is a specific form of exposure with response prevention (ERP), which is the treatment of choice for specific phobias, obsessive-compulsive disorder, and several other conditions. In flooding, clients are exposed to high-intensity stimuli, typically for a prolonged period of time, until their fear dissipates (Stern and Marks, 1973). For example, an individual with acrophobia (height phobia) might be taken to the top floor of a skyscraper and asked to look to the ground for several hours (flooding differs from another version of ERP, graded exposure, in which individuals are exposed to fear-inducing stimuli along a hierarchy of fear, from lowest to highest; Schumacher et al., 2015). Flooding can be performed "in vivo" (live), as in the aforementioned acrophobia example, imaginally, or via virtual reality. In contrast to generic flooding, implosion (implosive therapy) is a specific form of ERP in which individuals are imaginally exposed to extreme or grossly exaggerated versions of stimuli that ostensibly capture their "core" fear, which is presumed to derive from unconscious conflicts (Stampfl and Levis, 1968). For example, in implosion, the therapist might interpret a client's phobia of horses as symbolically reflecting 
unconscious Oedipal fears, and then systematically expose him to increasingly violent imagery of murdering his father.

(45) "Hypnagogic" versus "hypnopompic." Both terms refer to hallucinations that are often observed in individuals with narcolepsy, but that are reported from time to time in entirely normal individuals; such hallucinations are most often auditory but are sometimes visual instead or as well. Hypnagogic hallucinations occur as one is falling asleep, whereas hypnopompic hallucinations occur as one is coming out of sleep (Jones et al., 2009). As a handy mnemonic, recall that hypnagogic (note the italicized "g") hallucinations happen as one is going to sleep.

(46) "Insanity" versus "incompetence." Insanity is a legal term that bears on criminal responsibility. If defendants are deemed legally insane (at least in the 46 U.S. states in which the insanity defense remains on the books), they are not guilty of the crime. The basis for the not guilty by reason of insanity defense differs across states, but in most cases it hinges largely on whether the defendant understood what he or she was doing at the time of the crime, understood whether it was wrong, or both; some states also consider the defendant's ostensible ability to control his or her impulses. In contrast, if defendants are deemed to be incompetent to stand trial, they cannot face trial to begin with. Competency requires that the defendant understand the nature of the charges against him or her and be able to consult effectively with an attorney (Roesch, 1979; Johnson et al., 1990). Also commonly confused with legal insanity is diminished capacity, which refers to a legal judgment that extenuating circumstances (e.g., a defendant being under extreme personal stress at the time of a violent act) should lighten the defendant's punishment (Miller, 2015). In the case of diminished capacity, the defendant is deemed to be guilty but receives a lessened sentence.

(47) "Relapse" versus "recurrence." While we're at it, it is worth laying out the distinctions among the " $5 \mathrm{Rs"} \mathrm{of} \mathrm{psychological} \mathrm{and}$ psychopharmacological treatment: response, remission, recovery, relapse, and recurrence (Frank et al., 1991; Serani, 2011). Response refers to improvement in a psychological condition, especially depression, following a treatment; remission refers to full improvement that lasts at least 4 months following the treatment; and recovery refers to full improvement that lasts at least 6 months following the treatment. Two Rs that are especially easy to confuse are relapse and recurrence. The former refers to a return of the condition during the remission period (that is, prior to a recovery), whereas recurrence refers to a return of the condition following a recovery.

(48) "Stressor" versus "stress." Stressors refer to life events that have the potential to disrupt an organism's equilibrium, whereas stress refers to the organism's subjective reaction to stressors (Selye, 1956; Wheaton and Montazer, 2010). Hence, the titles of

\section{REFERENCES}

Akhtar, S. (2009). Comprehensive Dictionary of Psychoanalysis. London, UK: Karnac Books.

Ali, A. (2016). Schizophrenia, Symptoms, Types, Causes, and Treatment. OnHealth. Available at: http://www.onhealth.com/content/1/schizophrenia_treatment

American Psychiatric Association. (2013). Diagnostic and Statistical Manual of Mental Disorders, Fifth Edition (DSM-5). Washington, DC: American Psychiatric Association. such articles as "Does stress damage the brain?" (Bremner, 1999) are typically incorrect, as the studies reviewed therein focus largely on stressors as initial causes of brain damage rather than as mediators of brain damage.

(49) "Study" versus "experiment." A study refers to any kind of psychological investigation. In contrast, an experiment refers to a specific type of study in which participants are randomly assigned to groups and in which the researcher manipulates an independent variable (Mitchell and Jolley, 2012). Random assignment, incidentally, should not be confused with random selection, which refers to how participants were initially chosen, not to how they are allocated to experimental conditions (Sullivan, 2009).

(50) “Testing” versus “assessment.” Psychological testing refers to the act of administering psychological measures, such as selfreport indices, interviews, or intelligence tests, to individuals. In contrast, psychological assessment refers to the integration and interpretation of test scores, almost always in conjunction with other information (e.g., life history data, behavioral observations during testing) to draw inferences concerning the individual's mental status (Matarazzo, 1990; Hecker and Thorpe, 2005).

\section{CONCLUDING THOUGHTS}

The ability to distinguish between superficially different terms is a prerequisite for the acquisition of accurate scientific knowledge, including psychological knowledge. Without this capacity, conceptual confusion is virtually inevitable. More generally, several definitions of critical thinking in educational psychology underscore the necessity of acquiring a specialist vocabulary within one's domain of interest and even more important, making fine differentiations among seemingly related but conceptually distinct concepts (van Gelder, 2005). For example, one author team wrote that "acquiring critical concepts is not essentially a matter of acquiring new terminology; rather, it is a matter of learning to make appropriate distinctions" (Bailin et al., 1999, p. 293). In this vein, we hope that our "listicle" of frequently confused psychological term pairs will be a modest contribution toward enhancing not merely psychological literacy (Boneau, 1990) but also critical thinking in psychology more broadly.

\section{AUTHOR CONTRIBUTIONS}

SOL conceptualized and drafted the initial article and incorporated edits from coauthors. AP performed background research for the article and helped to identify terms. SJL assisted with writing several sections of the article and assisted with editing. RL and IW offered suggestions for terms and assisted with editing.

American Psychological Association. (2010). Publication Manual of the American Psychological Association. Washington, DC: American Psychological Association.

Anderson, C. A., and Bushman, B. J. (1997). External validity of "trivial" experiments: the case of laboratory aggression. Rev. Gen. Psychol. 1, 19-41. doi:10.1037/1089-2680.1.1.19

Asch, S. E. (1956). Studies of independence and conformity: I. A minority of one against a unanimous majority. Psychol. Monogr. 70, 1-70. doi:10.1037/h0093718 Baddeley, A. D. (1986). Working Memory. Oxford, UK: Oxford University Press. 
Bailin, S., Case, R., Coombs, J. R., and Daniels, L. B. (1999). Conceptualizing critical thinking. J. Curric. Stud. 31, 285-302. doi:10.1080/002202799183124

Baron, A., and Galizio, M. (2006). Distinguishing between positive and negative reinforcement: responses to Nakajima (2006) and Staats (2006). Behav. Anal. 29, 273-277. doi:10.1007/BF03392137

Baron, R. M., and Kenny, D. A. (1986). The moderator-mediator variable distinction in social psychological research: conceptual, strategic, and statistical considerations. J. Pers. Soc. Psychol. 51, 1173-1182. doi:10.1037/0022-3514.51.6.1173

Baron-Cohen, S. (2011). The Science of Evil: On Empathy and the Origins of Cruelty. New York, NY: Basic Books.

Bell, M., Milstein, R., Beam-Goulet, J., Lysaker, P., and Cicchetti, D. (1992). The positive and negative syndrome scale and the brief psychiatric rating scale: reliability, comparability, and predictive validity. J. Nerv. Ment. Dis. 180, 723-728. doi:10.1097/00005053-199211000-00007

BenDebba, M., Torgerson, W. S., and Long, D. M. (2000). A validated, practical classification procedure for many persistent low back pain patients. Pain 87 , 89-97. doi:10.1016/S0304-3959(00)00278-5

Ben-Zeev, A. (1984). The passivity assumption of the sensation-perception distinction. Br. J. Philos. Sci. 35, 327-343. doi:10.1093/bjps/35.4.327

Berg, J. M., Smith, S. F., Watts, A. L., Ammirati, R., Green, S. E., and Lilienfeld, S. O. (2013). Misconceptions regarding psychopathic personality: implications for clinical practice and research. Neuropsychiatry 3, 63-74. doi:10.2217/npy.12.69

Bleuler, E. (1911). Dementia praecox oder Gruppe der Schizophrenien, ed. G. Aschaffenburg (Leipzig, Deuticke: Handbuch der psychiatrie).

Bloom, P. (2017). Against Empathy. New York: HarperCollins.

Boneau, C. A. (1990). Psychological literacy: a first approximation. Am. Psychol. 45, 891-900. doi:10.1037/0003-066X.45.7.891

Bouton, M. E. (2004). Context and behavioral processes in extinction. Learn. Mem. 11, 485-494. doi:10.1101/lm.78804

Bremner, J. D. (1999). Does stress damage the brain? Biol. Psychiatry 45, 797-805. doi:10.1016/S0006-3223(99)00009-8

Campbell, D. T., and Fiske, D. W. (1959). Convergent and discriminant validation by the multitrait-multimethod matrix. Psychol. Bull. 56, 81-105. doi:10.1037/h0046016

Carmines, E. G., and Zeller, R. A. (1979). Reliability and Validity Assessment, Vol. 17. Newbury Park, CA: SAGE.

Centers for Educational Justice and Community Engagement. (2016). Definition of Terms. University of California at Berkeley, Division of Equity and Inclusion. Available at: http://ejce.berkeley.edu/geneq/resources/lgbtq-resources/ definition-terms

Chalasani, P., Healy, D., and Morriss, R. (2005). Presentation and frequency of catatonia in new admissions to two acute psychiatric admission units in India and Wales. Psychol. Med. 35, 1667-1675. doi:10.1017/S0033291705005453

Chiu, M. J., Chen, T. F., Yip, P. K., Hua, M. S., and Tang, L. Y. (2006). Behavioral and psychologic symptoms in different types of dementia. J. Formos. Med. Assoc. 105, 556-562. doi:10.1016/S0929-6646(09)60150-9

Clark, A. (2010). Empathy and sympathy: therapeutic distinctions in counseling. J. Mental Health Counsel. 32, 95-101. doi:10.17744/mehc.32.2.228n116thw 397504

Cleckley, H. (1941/1988). The Mask of Sanity. St. Louis, MO: Mosby.

Cohen, M. T. (2012). The importance of vocabulary for science learning. Kappa Delta Pi Rec. 48, 72-77. doi:10.1080/00228958.2012.680372

Cole, D. A. (1987). Utility of confirmatory factor analysis in test validation research. J. Consult. Clin. Psychol. 55, 584-594. doi:10.1037/0022-006X.55.4.584

Cornblatt, B., and Obuchowski, M. (1997). Update of high-risk research: 1987-1997. Int. Rev. Psychiatry 9, 437-447. doi:10.1080/09540269775303

Cortina, J. M. (1993). What is coefficient alpha? An examination of theory and applications. J. Appl. Psychol. 78, 98-104. doi:10.1037/0021-9010.78.1.98

Cowan, N. (2008). What are the differences between long-term, short-term, and working memory? Prog. Brain Res. 169, 323-338. doi:10.1016/S00796123(07)00020-9

Cranney, J., and Dunn, D. (eds) (2011). The Psychologically Literate Citizen: Foundations and Global Perspectives. New York: Oxford University Press.

Cronbach, L. J. (1951). Coefficient alpha and the internal structure of tests. Psychometrika 16, 297-334. doi:10.1007/BF02310555

Dauvilliers, Y., Arnulf, I., and Mignot, E. (2007). Narcolepsy with cataplexy. Lancet 369, 499-511. doi:10.1016/S0140-6736(07)60237-2

Decety, J., and Michalska, K. J. (2010). Neurodevelopmental changes in the circuits underlying empathy and sympathy from childhood to adulthood. Dev. Sci. 13, 886-899. doi:10.1111/j.1467-7687.2009.00940.x
Duckworth, K., Halpern, J. H., Schutt, R. K., and Gillespie, C. (2003). Use of schizophrenia as a metaphor in US newspapers. Psychiatr. Serv. 54, 1402-1404. doi:10.1176/appi.ps.54.10.1402

Fabrigar, L. R., Wegener, D. T., MacCallum, R. C., and Strahan, E. J. (1999). Evaluating the use of exploratory factor analysis in psychological research. Psychol. Methods 4, 272-299. doi:10.1037/1082-989X.4.3.272

Fox, J. A., and Levin, J. (1998). Multiple homicide: patterns of serial and mass murder. Crime Justice 23, 407-455. doi:10.1086/449274

Frances, A. (2013). Saving Normal: An Insider's Revolt Against Out-of-Control Psychiatric Diagnosis, DSM-5, Big Pharma and the Medicalization of Ordinary Life. New York: HarperCollins.

Frank, E., Prien, R. F., Jarrett, R. B., Keller, M. B., Kupfer, D. J., Lavori, P. W., et al. (1991). Conceptualization and rationale for consensus definitions of terms in major depressive disorder: remission, recovery, relapse, and recurrence. Arch. Gen. Psychiatry 48, 851-855. doi:10.1001/archpsyc.1991. 01810330075011

Frazier, P. A., Tix, A. P., and Barron, K. E. (2004). Testing moderator and mediator effects in counseling psychology research. J. Couns. Psychol. 51, 115-134. doi:10.1037/0022-0167.51.1.115

Freud, S. (1895). Project for a Scientific Psychology. London, UK: Hogarch Press.

Gay, P. (2006). Freud: A Life for Our Time. New York, NY: W.W. Norton \& Co.

Graham, K., and Wells, S. (2003). 'Somebody's gonna get their head kicked in tonight!': aggression among young males in bars-a question of values? $\mathrm{Br}$. J. Criminol. 43, 546-566. doi:10.1093/bjc/azg546

Gregoire, C. (2013). 6 Myths about Introverts to Stop Believing. Huffington Post. Available at: http://www.huffingtonpost.com/2015/09/01/introvert-myths_n 3569058.html

Gupta, S., and Shukla, A. P. (1989). Verbal operant conditioning as a function of extraversion and reinforcement. Br. J. Psychol. 80, 39-44. doi:10.1111/ j.2044-8295.1989.tb02302.x

Gurung, R. A. R., and Landrum, R. E. (2013). "Assessment and the scholarship of teaching and learning," in Assessing Teaching and Learning in Psychology: Current and Future Perspectives, eds D. Dunn, S. C. Baker, C. M. Mehrotra, R. E. Landrum, and M. McCarthy (Belmont, CA: Cengage), 159-171.

Hacking, I. (1995). Rewriting the Soul: Multiple Personality and the Sciences of Memory. Princeton, NJ: Princeton University Press.

Hale, C. R. (2002). Does multiculturalism menace? Governance, cultural rights and the politics of identity in Guatemala. J. Lat. Am. Stud. 34, 485-524. doi:10.1017/ S0022216X02006521

Hamstra, B. (1995). How Therapists Diagnose: Seeing Through the Psychiatric Eye. New York, NY: Macmillan.

Hare, R. D. (1991/2003). Manual for the Hare Psychopathy Checklist-Revised. Toronto, CA: Multihealth Systems.

Haynes, S. N., Richard, D., and Kubany, E. S. (1995). Content validity in psychological assessment: a functional approach to concepts and methods. Psychol. Assess. 7, 238-247. doi:10.1037/1040-3590.7.3.238

Haynes, S. N., Smith, G. T., and Hunsley, J. D. (2011). Scientific Foundations of Clinical Assessment. New York, NY: Taylor \& Francis.

Hecker, J. E., and Thorpe, G. L. (2005). Introduction to Clinical Psychology. Boston, MA: Allyn \& Bacon.

Henle, C. A. (2005). Predicting workplace deviance from the interaction between organizational justice and personality. J. Manage. Issues 17, 247-263.

Hoek, H. W., and Van Hoeken, D. (2003). Review of the prevalence and incidence of eating disorders. Int. J. Eat. Disord. 34, 383-396. doi:10.1002/eat. 10222

Hulme, C. (2014). Reading Retardation and Multi-sensory Teaching (Psychology Revivals). New York, NY: Routledge.

Hyman, R. (2017). "Pitfalls in parapsychological research," in Psychological Science under Scrutiny: Recent Challenges and Proposed Solutions, eds S. O. Lilienfeld and I. D. Waldman (New York: Wiley), 271-294.

James, L. R., and Brett, J. M. (1984). Mediators, moderators, and tests for mediation. J. Appl. Psychol. 69, 307-321. doi:10.1037/0021-9010.69.2.307

Johnson, W. G., Nicholson, R. A., and Service, N. M. (1990). The relationship of competency to stand trial and criminal responsibility. Crim. Justice Behav. 17, 169-185. doi:10.1177/0093854890017002002

Jones, S. R., Fernyhough, C., and Meads, D. (2009). In a dark time: development, validation, and correlates of the Durham hypnagogic and hypnopompic hallucinations questionnaire. Pers. Individ. Dif. 46, 30-34. doi:10.1016/j. paid.2008.08.021 
Keller, L. (1989). Convicted Mass Murderer Ted Bundy Showed Remove. Sun Sentinel. Available at: http://articles.sun-sentinel.com/1989-01-24/news/8901040963_ 1_colorado-investigators-execution-appeal

Kendler, K. S. (2005). Toward a philosophical structure for psychiatry. Am. J. Psychiatry 162, 433-440. doi:10.1176/appi.ajp.162.3.433

Kihlstrom, J. F. (2002). No need for repression. Trends Cogn. Sci. 6, 502. doi:10.1016/ S1364-6613(02)02006-5

Kleinman, A., Eisenberg, L., and Good, B. (2006). Culture, illness, and care: clinical lessons from anthropologic and cross-cultural research. Focus 4, 140-149. doi:10.1176/foc.4.1.140

Kölmel, H. W. (1993). Visual illusions and hallucinations. Baillieres Clin. Neurol. 2, 243-264.

Konnikova, M. (2016). The Psychological Research that Helps to Explain the Election. The New Yorker. Available at: http://www.newyorker.com/science/ maria-konnikova/the-psychological-research-that-helps-explain-the-election

Kraemer, H. C., Kazdin, A. E., Offord, D. R., Kessler, R. C., Jensen, P. S., and Kupfer, D. J. (1997). Coming to terms with the terms of risk. Arch. Gen. Psychiatry 54, 337-343. doi:10.1001/archpsyc.1997.01830160065009

Landgraf, S., Steingen, J., Eppert, Y., Niedermeyer, U., van der Meer, E., and Krueger, F. (2011). Temporal information processing in short-and long-term memory of patients with schizophrenia. PLoS ONE 6:e26140. doi:10.1371/ journal.pone.0026140

Laureys, S., Celesia, G. G., Cohadon, F., Lavrijsen, J., León-Carrión, J., Sannita, W. G., et al. (2010). Unresponsive wakefulness syndrome: a new name for the vegetative state or apallic syndrome. BMC Med. 8:68. doi:10.1186/1741-7015-8-68

Laureys, S., Owen, A. M., and Schiff, N. D. (2004). Brain function in coma, vegetative state, and related disorders. Lancet Neurol. 3, 537-546. doi:10.1016/ S1474-4422(04)00852-X

LeDoux, J. E., and Pine, D. S. (2016). Using neuroscience to help understand fear and anxiety: a two-system framework. Am. J. Psychiatry 173, 1083-1093. doi:10.1176/appi.ajp.2016.16030353

Levy, B. J., and Anderson, M. C. (2002). Inhibitory processes and the control of memory retrieval. Trends Cogn. Sci. 6, 299-305. doi:10.1016/ S1364-6613(02)01923-X

Lilienfeld, S. O. (1994). Conceptual problems in the assessment of psychopathy. Clin. Psychol. Rev. 14, 17-38. doi:10.1016/0272-7358(94)90046-9

Lilienfeld, S. O. (2004). Taking theoretical risks in a world of directional predictions. Appl. Prev. Psychol. 11, 47-51. doi:10.1016/j.appsy.2004.02.008

Lilienfeld, S. O., and Lynn, S. J. (2014). "Dissociative identity disorder: a contemporary scientific perspective," in Science and Pseudoscience in Clinical Psychology, 2nd Edn, eds S. O. Lilienfeld, S. J. Lynn, and J. M. Lohr (New York: Guilford Press), 113-152.

Lilienfeld, S. O., Lynn, S. J., Ruscio, J., and Beyerstein, B. L. (2009). Fifty Great Myths of Popular Psychology: Shattering Widespread Misconceptions about Human Behavior. Chichester, England: Wiley-Blackwell.

Lilienfeld, S. O., Sauvigné, K. C., Lynn, S. J., Cautin, R. L., Latzman, R. D., and Waldman, I. D. (2015). Fifty psychological and psychiatric terms to avoid: a list of inaccurate, misleading, misused, ambiguous, and logically confused words and phrases. Front. Psychol. 6:1100. doi:10.3389/fpsyg.2015.01100

Lilienfeld, S. O., Smith, S. F., and Watts, A. L. (2016). "Issues in diagnosis: conceptual issues and controversies," in Psychopathology: History, Diagnosis, and Conceptual Foundations, eds W. E. Craighead, D. J. Miklowitz, and L. W. Craighead (New York: Wiley), 1-26.

Lipowski, Z. J. (1990). Delirium: Acute Confusional States. New York: Oxford University Press.

Lippmann, S., and Perugula, M. L. (2016). Delirium or dementia? Innov. Clin. Neurosci. 13, 56-57.

Loevinger, J. (1987). Paradigms of Personality. New York: Freeman.

Lykken, D. T. (1995). The Antisocial Personalities. Hillsdale, NJ: Lawrence Erlbaum Associates, Inc.

Lynn, M. R. (1986). Determination and quantification of content validity. Nurs. Res. 35, 382-386. doi:10.1097/00006199-198611000-00017

Manjunatha, N., Saddichha, S., Sinha, B. N. P., and Khess, C. R. J. (2008). Assessment of mood and affect by mental state examination in different cultural contexts. Psychopathology 41, 336-337. doi:10.1159/000146072

Matarazzo, J. D. (1990). Psychological assessment versus psychological testing: validation from Binet to the school, clinic, and courtroom. Am. Psychol. 45, 999-1017. doi:10.1037/0003-066X.45.9.999
Mayou, R. (2014). Is the DSM-5 chapter on somatic symptom disorder any better than DSM-IV somatoform disorder? Br. J. Psychiatry 204, 418-419. doi:10.1192/ bjp.bp. 113.134833

McCann, B. S., Scheele, L., Ward, N., and Roy-Byrne, P. (2000). Discriminant validity of the Wender Utah Rating Scale for attention-deficit/hyperactivity disorder in adults. J. Neuropsychiatry Clin. Neurosci. 12, 240-245. doi:10.1176/jnp.12.2.240

McGovern, T. V., Corey, L. A., Cranney, J., Dixon, W. E. Jr., Holmes, J. D., Kuebli, J. E., et al. (2010). "Psychologically literate citizens," in Undergraduate Education in Psychology: Blueprint for the Discipline's Future, ed. D. Halpern (Washington, DC: American Psychological Association), 9-27.

McKenzie, D. (2014). The Near Death Experience: A Clinical Investigation. Available at: http://www.lulu.com/shop/duncan-alexander-mckenzie-rn/the-near-deathexperience-a-clinical-investigation/paperback/product-21745863.html

Mealey, L. (1995). The sociobiology of sociopathy: an integrated evolutionary model. Behav. Brain Sci. 18, 523-541. doi:10.1017/S0140525X00039595

Meehl, P. E. (1954). Clinical versus Statistical Prediction: A Theoretical Analysis and a Review of the Evidence. Minneapolis, MN: University of Minnesota Press.

Michelson, S. (2011). British Soccer Players Get Negative Reinforcement. ABC News. Available at: http://abcnews.go.com/International/story?id=80629

Milgram, S. (1963). Behavioral study of obedience. J. Abnorm. Soc. Psychol. 67, 371-378. doi:10.1037/h0040525

Miller, L. (2015). "PTSD in the criminal justice system II: the insanity defense and diminished capacity," in PTSD and Forensic Psychology, ed. L. Miller (New York: Springer International Publishing), 69-81.

Mitchell, M. L., and Jolley, J. M. (2012). Research Design Explained. Belmont, CA: Cengage Learning.

Monti, M. M., Laureys, S., and Owen, A. M. (2010). The vegetative state. Br. Med. J. 341, 292-296. doi:10.1136/bmj.c3765

Mook, D. G. (1983). In defense of external invalidity. Am. Psychol. 38, 379-387. doi:10.1037/0003-066X.38.4.379

Morrison, J. (2014). DSM-5 $5^{\circledR}$ Made Easy: The Clinician's Guide to Diagnosis. New York: Guilford Publications.

Mystkowski, J. L., Craske, M. G., and Echiverri, A. M. (2002). Treatment context and return of fear in spider phobia. Behav. Ther. 33, 399-416. doi:10.1016/ S0005-7894(02)80035-1

Nevo, B. (1985). Face validity revisited. J. Educ. Meas. 22, 287-293. doi:10.1111/ j.1745-3984.1985.tb01065.x

Owen, A. M., Coleman, M. R., Boly, M., Davis, M. H., Laureys, S., and Pickard, J. D. ( 2006). Detecting awareness in the vegetative state. Science 313, 1402-1402. doi:10.1126/science.1130197

Partridge, G. E. (1930). Current conceptions of psychopathic personality. Am. J. Psychiatry 87, 53-99. doi:10.1176/ajp.87.1.53

Patel, N. C., DelBello, M. P., Keck, P. E., and Strakowski, S. M. (2006). Phenomenology associated with age at onset in patients with bipolar disorder at their first psychiatric hospitalization. Bipolar Disord. 8, 91-94. doi:10.1111/j.1399-5618.2006.00247.x

Patterson, G. R., Reid, J. B., and Dishion, T. J. (1992). Antisocial Boys: A Social Interactional Approach, Vol. 4. Eugene, OR: Castalia.

Pemment, J. (2013). Psychopathy versus sociopathy: why the distinction has become crucial. Aggress. Violent Behav. 18, 458-461. doi:10.1016/j.avb.2013.07.001

Rieder, E., and Tausk, F. (2012). Psoriasis, a model of dermatologic psychosomatic disease: psychiatric implications and treatments. Int. J. Dermatol. 51, 12-26. doi:10.1111/j.1365-4632.2011.05071.x

Roberts, L. D., Heritage, B., and Gasson, N. (2015). The measurement of psychological literacy: a first approximation. Front. Psychol. 6:105. doi:10.3389/ fpsyg.2015.00105

Robins, L. N. (1966). Deviant Children Grown Up. Baltimore, MD: Williams \& Wilkins.

Roesch, R. (1979). Determining competency to stand trial: an examination of evaluation procedures in an institutional setting. J. Consult. Clin. Psychol. 47, 542-550. doi:10.1037/0022-006X.47.3.542

Schmader, T., and Lickel, B. (2006). The approach and avoidance function of guilt and shame emotions: comparing reactions to self-caused and other-caused wrongdoing. Motiv. Emot. 30, 42-55. doi:10.1007/s11031-006-9006-0

Schumacher, S., Miller, R., Fehm, L., Kirschbaum, C., Fydrich, T., and Ströhle, A. (2015). Therapists' and patients' stress responses during graduated versus flooding in vivo exposure in the treatment of specific phobia: a preliminary observational study. Psychiatry Res. 230, 668-675. doi:10.1016/j.psychres.2015.10.020 
Schwartz, B. M., Landrum, R. E., and Gurung, R. A. (2016). An Easy Guide to APA Style. New York: SAGE.

ScienceDaily. (2011). Everyday 'Clairvoyance': How Your Brain Makes Near-Future Predictions. Washington University. Available at: https://www.sciencedaily. com/releases/2011/08/110817175925.htm

Selye, H. (1956). The Stress of Life. New York, NY: McGraw-Hill.

Serani, D. (2011). Depression: Do You Know All Your R's? Psychology Today Blogs. Available at: https://www.psychologytoday.com/blog/two-takes-depression/ 201103/depression-do-you-know-all-your-rs

Siciliano, R. (2014). Psychopath vs. Sociopath: What's the Difference? Huffington Post. Available at: http://www.huffingtonpost.com/robert-siciliano/what-is-asociopath_b_5877160.html

Sijtsma, K. (2009). On the use, the misuse, and the very limited usefulness of Cronbach's alpha. Psychometrika 74, 107-120. doi:10.1007/s11336-008-9101-0

Sink, K. M., Holden, K. F., and Yaffe, K. (2005). Pharmacological treatment of neuropsychiatric symptoms of dementia: a review of the evidence. J. Am. Med. Assoc. 293, 596-608. doi:10.1001/jama.293.5.596

Smith, R. H., and Kim, S. H. (2007). Comprehending envy. Psychol. Bull. 133, 46-64. doi:10.1037/0033-2909.133.1.46

Stampfl, T. G., and Levis, D. J. (1968). Implosive therapy-a behavioral therapy? Behav. Res. Ther. 6, 31-36. doi:10.1016/0005-7967(68)90039-9

Starr, L. R., Hammen, C., Connolly, N. P., and Brennan, P. A. (2014). Does relational dysfunction mediate the association between anxiety disorders and later depression? Testing an interpersonal model of comorbidity. Depress. Anxiety 31, 77-86. doi:10.1002/da.22172

Steinkamp, F. (1999). Testing clairvoyance and precognition by manipulating probabilities: a conceptual assessment of the experimental literature. J. Parapsychol. 63, 99-130.

Stern, G. S., McCants, T. R., and Pettine, P. W. (1982). Stress and illness controllable and uncontrollable life events' relative contributions. Pers. Soc. Psychol. Bull. 8 , 140-145. doi:10.1177/014616728281022

Stern, R., and Marks, I. (1973). Brief and prolonged flooding: a comparison in agoraphobic patients. Arch. Gen. Psychiatry 28, 270-276. doi:10.1001/ archpsyc. 1973.01750320098015

Sternberg, R. J., and Sternberg, K. (2010). The Psychologist's Companion: A Guide to Writing Scientific Papers for Students and Researchers. New York: NY: Cambridge University Press.

Streiner, D. L. (1996). Maintaining standards: differences between the standard deviation and standard error, and when to use each. Can. J. Psychiatry 41, 498-502. doi:10.1177/070674379604100805

Streiner, D. L. (1998). Let me count the ways: measuring incidence, prevalence, and impact in epidemiological studies. Can. JPsychiatry 43, 173-179. doi:10.1177/070674379804300207

Streiner, D. L. (2013). "Regression in the service of the superego: the dos and don'ts of stepwise multiple regression," in A Guide for the Statistically Perplexed: Selected Readings for Clinical Researchers, ed. D. L. Streiner, (Toronto, CA: University of Toronto Press), 123-136.

Sullivan, L. E. (2009). "Random assignment vs. random selection," in The SAGE Glossary of the Social and Behavioral Sciences. Available at: http://sk.sagepub. com/reference/behavioralsciences/n2113.xml

Sylvers, P., Lilienfeld, S. O., and LaPrairie, J. L. (2011). Differences between trait fear and trait anxiety: implications for psychopathology. Clin. Psychol. Rev. 31, 122-137. doi:10.1016/j.cpr.2010.08.004

Tangney, J. P. (1996). Conceptual and methodological issues in the assessment of shameandguilt.Behav.Res. Ther.34,741-754.doi:10.1016/0005-7967(96)00034-4
Tauber, R. T. (1988). Overcoming misunderstanding about the concept of negative reinforcement. Teach. Psychol. 15, 152-153. doi:10.1207/s15328023top1503_15

Tedeschi, J. T., and Quigley, B. M. (1996). Limitations of laboratory paradigms for studying aggression. Aggress. Violent Behav. 1, 163-177. doi:10.1016/1359-1789(95)00014-3

van Gelder, T. (2005). Teaching critical thinking: some lessons from cognitive science. Coll. Teach. 53, 41-48. doi:10.3200/CTCH.53.1.41-48

Van Zandwijk, N., and Van De Vijver, M. J. (2007). Clairvoyance or reliable prediction of the future? Ann. Oncol. 18, 407-408. doi:10.1093/annonc/ mdl503

Veletsianos, G., and Russell, G. S. (2014). "Pedagogical agents," in Handbook of Research on Educational Communications and Technology, eds J. M. Spector, M. D. Merrill, J. Elen, and M. J. Bishop (New York, NY: Springer New York), 759-769.

Waldman, I. D., Lilienfeld, S. O., and Lahey, B. B. (1995). "Toward construct validity in the childhood disruptive behavior disorders," in Advances in Clinical Child Psychology, eds T. H. Ollendick and R. J. Prinz (New York, NY: Springer), 323-363.

Weiser, M., Knobler, H. Y., Noy, S., and Kaplan, Z. (2002). Clinical characteristics of adolescents later hospitalized for schizophrenia. Am. J. Med. Genet. 114, 949-955. doi:10.1002/ajmg.10647

Welsh, D. T., and Ordóñez, L. D. (2014). Conscience without cognition: the effects of subconscious priming on ethical behavior. Acad. Manage. J. 57, 723-742. doi:10.5465/amj.2011.1009

Wheaton, B., and Montazer, S. (2010). "Stressors, stress, and distress," in A Handbook for the Study of Mental Health: Social Contexts, Theories, and Systems, eds T. L. Scheid and T. N. Brown (Cambridge, UK: Cambridge University Press), 171-199.

White, T. L., and Depue, R. A. (1999). Differential association of traits of fear and anxiety with norepinephrine- and dark-induced pupil reactivity. J. Pers. Soc. Psychol. 77, 863-877. doi:10.1037/0022-3514.77.4.863

Whittingham, M. J., Stephens, P. A., Bradbury, R. B., and Freckleton, R. P. (2006). Why do we still use stepwise modelling in ecology and behaviour? J. Anim. Ecol. 75, 1182-1189. doi:10.1111/j.1365-2656.2006.01141.x

Wite, E. H. (1987). Behaviour in group situations: an integrative model. Eur. J. Soc. Psychol. 17, 403-429. doi:10.1002/ejsp.2420170403

Wolpe, J. (1987). "The promotion of scientific psychotherapy: a long voyage," in The Evolution of Psychotherapy, ed. J. K. Zeig (New York: Brunner/Mazel), $133-142$.

Yager, R. E. (1983). The importance of terminology in teaching K-12 science. J. Res. Sci. Teach. 20, 577-588. doi:10.1002/tea.3660200610

Zaki, J. (2014). Empathy: a motivated account. Psychol. Bull. 140, 1608-1647. doi:10.1037/a0037679

Conflict of Interest Statement: The authors declare that the research was conducted in the absence of any commercial or financial relationships that could be construed as a potential conflict of interest.

Copyright (C) 2017 Lilienfeld, Pydych, Lynn, Latzman and Waldman. This is an open-access article distributed under the terms of the Creative Commons Attribution License (CC BY). The use, distribution or reproduction in other forums is permitted, provided the original author(s) or licensor are credited and that the original publication in this journal is cited, in accordance with accepted academic practice. No use, distribution or reproduction is permitted which does not comply with these terms. 